\title{
Efecto del cambio climático en la producción de semilla de zámota (Coursetia glandulosa A. Gray)
}

\author{
Effect of climate change on the production of zamota (Coursetia glandulosa) seed \\ Diana M. Mc Caughey-Espinoza ${ }^{1}$, Nydia E. Buitimea-Cantúa ${ }^{2 *}$, Gloria Irma Ayala-Astorga ${ }^{1}$, \\ Andrés Ochoa-Meza³, María G. Burboa-Zazueta ${ }^{1}$, Luis E. Gutiérrez-Millán ${ }^{1}$
}

\begin{abstract}
RESUMEN
La zámota (Coursetia glandulosa A. Gray) es una planta no maderable, que puede llegar a medir más de 3 metros de altura. Es importante para el sector agropecuario en el estado de Sonora, México, pero en la actualidad está presentando serios problemas derivados de los efectos del cambio climático. El objetivo de este trabajo fue determinar el efecto del calentamiento global en la producción de semillas de zámota en su hábitat natural. En la producción de semillas se observaron diferencias significativas $(P<0,05)$, de acuerdo a la época de colecta y extractos evaluados (altura de plantas: 1,5 a 1,80; 1,90 a 2,20 m). En el 2019 se obtuvo una producción total de semillas de 14,87 kg entre ambos extractos, mientras que la producción para el 2020 fue de 6,34 kg de semillas colectadas. Por lo tanto, la producción de semillas en el 2019 fue 2,34 veces mayor que la alcanzada en el 2020. En el 2019, el E2 obtuvo un promedio de producción de semillas por planta de $0,598 \mathrm{~g}$ con una desviación estándar de 0,052 y para el E1 fue de $0,393 \mathrm{~g}$ de semillas con una desviación estándar de 0,022, mientras que para el 2020, el E2 presentó un promedio de 0,253 g y el E1 fue de 0,169 g. En cuanto a las temperaturas máximas, mínimas y humedad relativa evaluadas en ambos periodos, su comportamiento fue similar, excepto la precipitación pluvial, que fue más baja en el 2020, sobre todo en la época más crítica del año que es verano.
\end{abstract}

Palabras clave: Cambio climático, Cousetia glandulosa, semillas, zámota.

\section{ABSTRACT}

The zamota (Coursetia glandulosa A. Gray) is a non-timber plant, which can measure up to approximately 3 meters, it is important for the agricultural sector in the state of Sonora, currently it is presenting serious problems derived from the effects negative produced by climate change. The objective of this work was to determine the effect of global warming on the production of Zamota seeds in their natural habitat. In seed production, there were significant differences $(P<0.05)$, according to the collection time and extracts evaluated (plant height: 1.5 to $1.80 ; 1.90$ to $2.20 \mathrm{~m}$ ). A total seed production was obtained in 2019 of $14.87 \mathrm{~K}$ of seeds between both extracts, while the production for 2020 was 6.34 K of collected seeds. Therefore, the seed production in 2019 was 2.34 times higher than that obtained in 2020. In 2019, the E2 presented an average seed production per plant of $0.598 \mathrm{~g}$ with a standard deviation of 0.052 and for the E1 it was of $0.393 \mathrm{~g}$ of seeds with a standard deviation of 0.022, while for 2020, the E2 presented an average of $0.253 \mathrm{~g}$ and the E1 was $0.169 \mathrm{~g}$. The maximum, minimum temperatures and relative humidity evaluated in both years, their behavior was similar except the rainfall was lower in 2020, especially in the most critical time of the year, which is summer.

Keywords: Climate change, Cousetia glandulosa, seeds, zamota.

\section{Introducción}

El estado de Sonora comprende una extensión territorial de $180605 \mathrm{~km}^{2}$ (INEGI, 2000). Las actividades de importancia socioeconómica son la agricultura, ganadería, pesca, acuacultura y minería.
Esta última ha sido uno de los sectores que en los últimos 15 años han detonado una demanda económica significativa en el estado. Según datos de la Comisión Nacional Forestal (CONAFOR), a nivel país, Sonora tiene el primer lugar en la deforestación y cada año pierde 24 mil 980,48 hectáreas de ecosistemas

1 Departamento de Investigaciones Científicas y Tecnológicas de la Universidad de Sonora. Blvd. Hermosillo, Sonora, México.

2 Tecnológico de Monterrey, Centro de Biotecnología-FEMSA, Escuela de Ingeniería y Ciencias, Campus Monterrey. Monterrey, N.L., México.

3 Departamento de Agricultura y Ganadería de la Universidad de Sonora. Hermosillo, Sonora.

* Autor de correspondencia: nebc@tec.mx 
naturales (Padilla, 2018). Esta drástica reducción de la superficie forestal acelera el cambio climático e induce a la desfragmentación de la biodiversidad aun existente y que en estos últimos años ha sido más vulnerable a sufrir alteraciones importantes como la pérdida de ecosistemas naturales.

La destrucción de áreas forestales ocasiona graves problemas ecológicos como la baja autopropagación de las especies nativas y la falta de alimento para animales herbívoros y aves. Por lo tanto, la producción de semillas forestales es deficiente. Existen especies cuyas poblaciones han disminuido entre 30 y $70 \%$ como palo fierro (Olneya tesota A. Gray), cosahui (Krameria erecta Willd), guayacán (Guaiacum coulteri), mezquite (Prosipis juliflora), palo blanco (Ipomoea arborescens) y zámota (Coursetia glandulosa A. Gray), las cuales pueden desaparecer de los ecosistemas (Mc Caughey-Espinoza et al., 2017). Por otra parte, investigadores han concluido que a nivel mundial el cambio climático pudiera tener un impacto catastrófico en la distribución de algunas especies arbóreas, con una disminución del $73 \%$ al $100 \%$ para finales de siglo (García-Valdez y Morales-Castilla, 2016).

Es importante buscar nuevas alternativas que permitan mejorar las áreas perturbadas rehabilitando los ecosistemas para generar y preservar la flora y fauna silvestre, y así minimizar los efectos del calentamiento global (Mc Caughey-Espinoza et al., 2019). Desafortunadamente, la disponibilidad de semilla de especies forrajeras es limitada por las bajas precipitaciones y esto repercute en la cantidad y calidad de la semilla de plantas arbóreas producidas en agostaderos (Martin et al., 2016). Por lo tanto, la conservación de los ecosistemas y la colecta de semillas para la reforestación con plantas nativas es la manera más idónea de contrarrestar el calentamiento global.

El objetivo de este trabajo fue determinar el efecto de los cambios en las temperaturas, el porcentaje de humedad relativa y la precipitación pluvial como consecuencia del calentamiento global, en la producción de semillas de zámota (Coursetia glandulosa A. Gray) en su hábitat natural. Las semillas fueron monitoreadas durante dos años.

\section{Materiales y métodos}

\section{Área de estudio}

La investigación se llevó a cabo en el Rancho Bella Vista, que se encuentra en las coordenadas $29^{\circ} 10^{\prime} 02.83^{\prime \prime}$ Norte y los $110^{\circ} 58^{\prime} 47.48^{\prime \prime}$ Oeste, con una altitud de $277 \mathrm{msnm}$, precipitación media anual de $330 \mathrm{~mm}$ y temperatura promedio de $24^{\circ} \mathrm{C}$. Tiene $\mathrm{x}$ un tipo de suelo regosol y su vegetación dominante es un matorral arbosufrutescente (COTECOCA, 2002; INEGI, 2007; SAGARPA, 2010).

\section{Especie en estudio}

Para este estudio se evaluó la producción de semillas de zámota (Coursetia glandulosa A. Gray), por ser un arbusto no maderable que proporciona forraje en épocas críticas del año a animales herbívoros. Es una planta forrajera clave del desierto de Sonora, México (Arellano et al., 2011; USDA-NRCS, 2015; Mc Caughey-Espinoza et al., 2017).

\section{Selección de arbustos}

Se consideraron 15 plantas adultas (fisiológicamente) por extracto para la evaluación de producción de semillas con una altura de 1,5 a 1,80 y de 190 a 2,20 metros.

\section{Colecta de semillas}

Este arbusto produce frutos dehiscentes y no se cubrieron las plantas para tener las condiciones naturales. La cosecha se realizó en el mes de mayo de 2019 y 2020.

\section{Variable a evaluar}

Producción de semillas: para la realización de las mediciones se utilizaron dos extractos de altura de plantas de 1,5 a 1,80 (primer extracto, E1) y de 1,90 a 2,20 metros (segundo extracto, E2). Se evaluó la producción de semillas en su hábitat natural. Se utilizó una balanza digital Pelouze, Modelo SP5, para pesar la producción de semilla en estudio.

Para determinar los efectos del cambio climático en nuestra región se monitoreó el comportamiento climatológico de algunos parámetros en promedio anual, de temperaturas máximas y mínimas, y porcentaje de humedad relativa, utilizando un medidor y registrador Data Logger Modelo WK057. También se midió la precipitación pluvial con un pluviómetro. Estas evaluaciones se llevaron a cabo durante el periodo de 2019 a 2020. 


\section{Análisis estadístico}

Para determinar si existen diferencias significativas con respecto a la producción de semillas se utilizó un diseño completamente al azar. Se realizó un análisis de varianza (ANDEVA) con un nivel de significancia de $P<0,05$, y una comparación de medias por Tukey. Para el análisis de datos se empleó el programa estadístico JMP versión 9.0.1 (JMP, 2011).

\section{Resultados y discusión}

Al evaluar la producción de semillas en la época de colecta y los extractos de altura (1,5 a $1,80 ; 1,90$ a $2,20 \mathrm{~m})$ se encontraron diferencias significativas $(P<0,05)$. En el 2019 se obtuvo una producción de $14,87 \mathrm{~kg}$ de semillas entre ambos extractos, mientras que la producción para el 2020 fue de 6,34 kg en total (Figura 1). Por lo tanto, la producción de semillas en el 2019 fue 2.34 veces mayor que la del 2020. En el año 2019 el E2 obtuvo un promedio de $0,598 \mathrm{~g}$ de semillas por planta con una desviación estándar de 0,052 y para el E1 fue de 0,393 g de semillas por planta con una desviación estándar de 0,022. En el 2020 el E2 presentó un promedio de $0,253 \mathrm{~g}$ de semillas y el E1 fue de $0,169 \mathrm{~g}$ de semillas. Sin embargo, una buena producción de semillas no asegura la propagación de las especies silvestres, ya que gran parte de estas son alimento para roedores y aves, aunado a la desertificación y falta de materia orgánica.

Para la evaluación de la producción de semillas se consideraron únicamente las semillas que estaban fisiológicamente maduras. Cabe mencionar que las bajas precipitaciones (\%), además de las altas

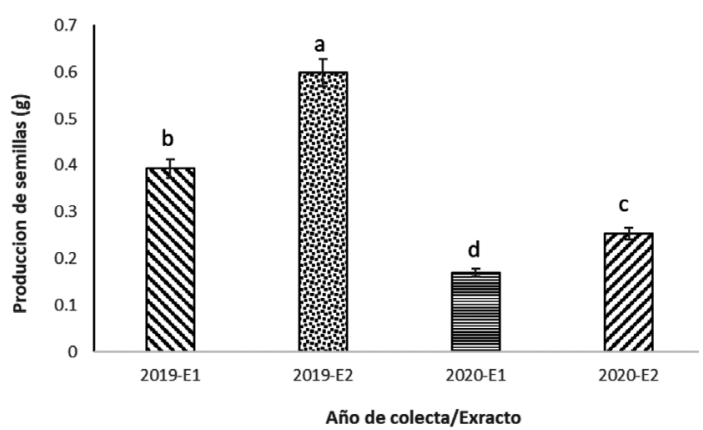

Figura 1. Producción de semillas de zámota colectada en los años 2019 y 2020 en diferentes extractos. temperaturas (valores) que se presentaron en el 2020, provocaron la disminución en la producción de semillas en las plantas de zámota. Por ello se prevé que a mediano plazo esta especie pueda presentar problemas en sus poblaciones silvestres a causa de los efectos acelerados del calentamiento global en los ecosistemas. En las Figuras 2 y 3 se muestra el impacto de las altas temperaturas en las vainas y semillas de zámota durante dos tiempos de evolución.

Es importante tomar en cuenta los efectos devastadores del cambio climático y que están afectando a los diferentes germoplasmas silvestres., Por consiguiente, es necesario adoptar medidas y trabajar para la preservación del patrimonio genético de las semillas y el desarrollo de especies (Chuet-Missé, 2017).

De igual manera se ve afectada la producción de semillas para la agricultura, pero con menor impacto. La pérdida de la biodiversidad provocada por los cambios en el medio ambiente nos obliga a realizar modificaciones a corto plazo en el estado de Sonora para la conservación de las semillas forestales.

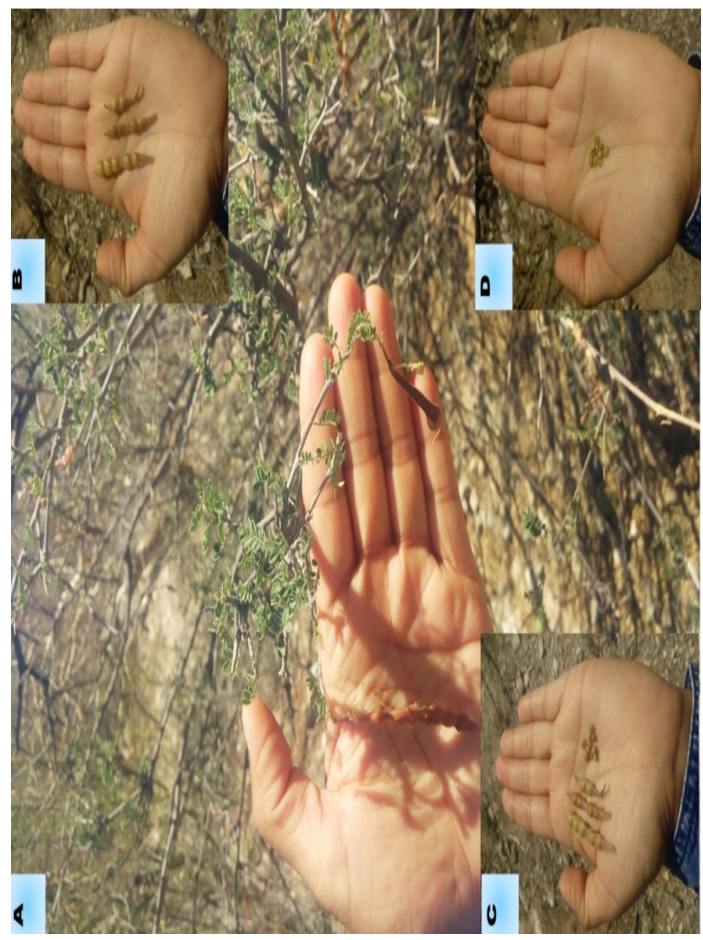

Figura 2. A: Zámota en su etapa de producción de semillas, B y C: Vainas inmaduras D: semillas maduras (2019). 


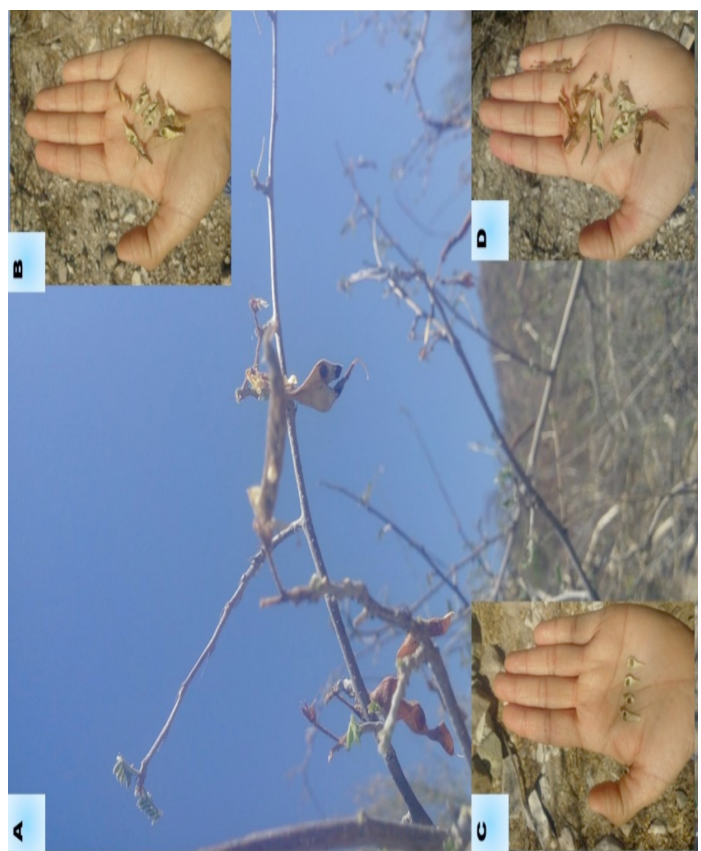

Figura 3. A: Zámota en su etapa de producción de semillas, B y C: Vainas inmaduras D: semillas inmaduras (2020).

Estas condiciones climáticas han sido catastróficas para la producción de semillas de zámota y posiblemente también para otras especies de importancia forrajera en la región. Es necesario señalar que existen grupos de personas dedicadas a la colecta de semillas para su venta, como una forma de sustento para sus familias. El kilo de semillas de zámota tiene un precio promedio regional de \$5,000.00 M.N. (pesos mexicanos) y dependiendo de la cantidad de recolectores se paga \$140 diarios por persona (Vallentine, 1980; Granite Seed Co., 2014).

Martín-Rivera et al. (2016) reportaron que se obtiene una producción de semilla de zámota de 0,85 a $1,1 \mathrm{~kg}$ por planta. Una persona colecta en promedio $0,195 \mathrm{~kg}$ diarios de semillas. Esta producción de semilla de zámota difiere de los resultados obtenidos en este estudio en el 2019 $(0,393$ a 0,598 g) y $2020(0,159$ a 0,253 g), y ese comportamiento puede atribuirse a las bajas precipitaciones registradas durante la época de colecta de las semillas. En el 2016 se registró una precipitación media de $(\sim 320 \mathrm{~mm})$, que fue suficiente para que las plantas estuvieran fisiológicamente vigorosas para la producción de flor y por ende para la producción de semillas (Martín-Rivera et al., 2016).
La planta de zámota es un arbusto perenne. También se considera uno de los principales forrajes en el desierto de Sonora, porque al contener un promedio de $20 \%$ de proteína cruda, es muy palatable para los animales herbívoros. La zámota es una planta sin espinas, frondosa en verano, y en esta época critica del año los animales consumen la flor y vaina (Arellano et al., 2011; USDA-NRCS, 2015), lo que constituye un factor limitante para su autopropagación. Sánchez et al. (2011) midieron la viabilidad de semillas de zámota (Coursetia glandulosa) con respecto al tiempo de almacenamiento $(0,25 ; 1,25 ; 2,25 ; 3,25$ o 4,25 años) y el efecto de la temperatura $\left(25,12\right.$ y $\left.-4^{\circ} \mathrm{C}\right)$ sobre la longevidad, y concluyeron que el mayor porcentaje de germinación fue en el tiempo de 0,25 años con $96,5 \%$, reduciéndose su germinación hasta $0,0 \%$ durante su almacenamiento. Con respecto a la longevidad, las semillas que fueron almacenadas a $-4{ }^{\circ} \mathrm{C}$ presentaron un $76 \%$ de germinación. Las semillas que permanecieron almacenadas en condiciones ambientales mostraron un $48.8 \%$ de germinación. Estos resultados obtenidos por Arellano et al. (2011) evidencian que las semillas de zámota son muy susceptibles a los cambios ambientales al perder su capacidad de maduración dentro de la vaina, como también el efecto negativo que provoca en el embrión.

El sector ganadero se ve afectado por las sequías prolongadas, y esto se manifiesta en el bajo peso del ganado, muerte o abandono de los agostaderos a causa de la variación de las condiciones climatológicas a nivel mundial. Es importante tener en cuenta que la producción y calidad de las semillas de las especies silvestres depende de la presencia de lluvias, y que los fenómenos climáticos, como temperatura, patrones de precipitación, nivel del mar, nubosidad, son afectados por el cambio en el clima del planeta (Prentice, 2001).

De acuerdo a las mediciones climatológicas (Tabla 1), se observa que en los años evaluados las temperaturas máximas y mínimas, así como la humedad relativa, fueron similares. Sin embargo, hubo diferencias en la precipitación pluvial, con precipitaciones más bajas en el 2020, en la época más crítica del año (verano), lo que conllevó la merma de la producción de semillas. Hammermeister (2000) menciona que las plantas tienden a disminuir su producción de semillas de acuerdo con las condiciones climáticas de 
Tabla 1. Promedio mensual de las condiciones climatológicas en los años 2019 y 2020.

\begin{tabular}{lcccc}
\hline Mes/año & Temp Máx $\left({ }^{\circ} \mathrm{C}\right)$ & Temp Min $\left({ }^{\circ} \mathrm{C}\right)$ & PP $(\mathrm{mm})$ & $\% \mathrm{HR}$ \\
\hline Enero 2019 & 25,58 & 8,81 & 0,77 & 52,48 \\
Enero 2020 & 25,45 & 8,39 & 0,94 & 58,19 \\
Febrero 2019 & 24,61 & 9,75 & 0,18 & 49,25 \\
Febrero 2020 & 27,00 & 10,21 & 0,66 & 45,41 \\
Marzo 2019 & 29,87 & 12,52 & 0,00 & 39,19 \\
Marzo 2020 & 27,65 & 12,65 & 0,00 & 55,35 \\
Abril 2019 & 33,00 & 17,27 & 0,00 & 29,43 \\
Abril 2020 & 33,10 & 15,37 & 0,00 & 27,47 \\
Mayo 2019 & 36,71 & 16,74 & 0,07 & 34,87 \\
Mayo 2020 & 38,65 & 21,26 & 0,00 & 23,23 \\
Junio 2019 & 41,20 & 21,70 & 0,07 & 33,90 \\
Junio 2020 & 41,63 & 25,57 & 0,10 & 24,83 \\
Julio 2019 & 40,61 & 27,00 & 7,94 & 43,13 \\
Julio 2020 & 41,74 & 29,32 & 0,65 & 40,84 \\
Agosto 2019 & 39,90 & 27,23 & 8,68 & 55,55 \\
Agosto 2020 & 40,1 & 29,29 & 0,81 & 44,68 \\
Septiembre 2019 & 34,90 & 25,43 & 4,47 & 66,53 \\
Septiembre 2020 & 39,63 & 27,17 & 0,00 & 39,93 \\
Octubre 2019 & 30,10 & 21,77 & 1,94 & 45,71 \\
Octubre 2020 & 38,16 & 21,03 & 0,00 & 32,35 \\
Noviembre 2019 & 26,97 & 18,40 & 1,87 & 57,60 \\
Noviembre 2020 & 25,10 & 5,03 & 0,00 & 54,21 \\
Diciembre 2019 & 22,10 & 13,23 & 1,39 & 61,61 \\
Diciembre 2020 & 29,56 & 4,11 & 0,13 & 57,35 \\
\hline
\end{tabular}

Temp Máx = Temperatura máxima, Temp Min = Temperatura mínima, ${ }^{\circ} \mathrm{C}=$ Grados Centígrados, $\mathrm{PP}(\mathrm{mm})=$ Precipitación Pluvial en mm y \% HR = Porciento de Humedad Relativa.

los agostaderos, los cuales pueden presentar temporadas secas o bajas precipitaciones. Por lo tanto, algunas plantas pueden adelantar o atrasar su producción o simplemente no producen semillas (Jorgensen y Stevens, 2004; Ibarra et al., 2009). Para minimizar los efectos de la baja producción de semillas, se han realizado estudios en México mediante la evaluación de diferentes climas y especies forrajeras, para producir semillas de buena o excelente calidad bajo condiciones de temporal o riego con una adecuada fertilización (Cuellar y Hernández, 2007; Herrera, 2008). En agostaderos con acceso al agua se pueden obtener semillas de calidad (Jorgensen y Stevens, 2004). Por ello es fundamental seguir monitoreando el efecto del cambio climático en la producción de los recursos fitogenéticos de las plantas nativas del desierto sonorense. En regiones áridas la vida de las especies depende, ecológica y socioeconómicamente, de las precipitaciones que se presenten.

La productividad de las plantas depende de la cantidad de agua y la eficiencia del organismo que la utiliza. La falta de agua puede limitar el crecimiento y productividad de las plantas. Dentro de la aclimatación al déficit hídrico, se encuentran la inhibición de la expansión foliar, el aumento de la profundización de las raíces, abscisión foliar y cierre estomático. Entre las actividades dependientes de la turgencia celular, la expansión foliar y el alongamiento radicular son las más sensibles al déficit hídrico. Parmesan (2006) mostró evidencias sobre muchos taxones que respondieron al calentamiento global dispersándose hacia elevaciones mayores. Este fenómeno se presenta, con ciertas alteraciones (Wilson et al., 2007; Moritz et al., 2008), sobre todo en las plantas leñosas (Jump et al., 2009; Yepes y Buckeridge, 2011).

\section{Conclusiones}

Los altos índices de deforestación que se registran en el estado de Sonora, México, podrían acelerar la disminución de zámota (Coursetia glandulosa A. Gray) en sus ecosistemas. Esta es una planta que se encuentra en la categoría de especie rara en la Norma Oficial Mexicana.

En las regiones áridas la vida de las especies depende, ecológica y socioeconómicamente, de las precipitaciones que se presentan, aunado a las bajas precipitaciones pluviales. 
En consecuencia, es importante que las asociaciones ganaderas cuenten con bancos de germoplasmas con especies de mayor palatabilidad para el ganado. Así asegurarían también la perpetuidad de las especies. Además es recomendable implementar sus propios programas de reforestación en potreros o áreas de descanso (libres de pastoreo), para garantizar el establecimiento de las nuevas plantas. Todos tenemos la responsabilidad de seguir trabajando para desacelerar el proceso de desfragmentación genética que ocurre a nivel global producto del cambio climático.

\section{Agradecimientos}

A Yeimi C. Mc Caughey Espinoza, por su gran ayuda en las salidas al campo y colecta de semillas.

\section{Literatura Citada}

Arellano, J.G.S.; Parra-Galindo, M.A.; Silva-Olivas, M.F.; Pedroza-Pérez, D.

2011. Efecto de la temperatura y tiempo de almacenamiento sobre la viabilidad en semillas de zámota (Coursetia glandulosa, Gray). Revista Biotecnia, 13(3): 36-40.

Chuet-Missé J.P.

2017. Las súper semillas que resisten el cambio climático. Periódico La Vanguardia. https://www.lavanguardia.com/ natural/20170228/42358632446/semillas-cambio-climatico. html Consultado: 12/ago/2020.

COTECOCA.

2002. Diagnóstico de los agostaderos del Estado de Sonora. SAGARPA. Coordinación General de Ganadería. México. $52 \mathrm{p}$.

Cuellar, V.E.J.; Hernández, R.P.

2007. Producción de semilla de pasto buffel Zaragoza 115 con aplicación de nitrógeno, fósforo y potasio en el norte de Coahuila. Resumen. XLIII Reunión Nacional de Investigación Pecuaria. Culiacán.

García-Valdés, R.; Morales-Castilla, I.

2016. Efectos del cambio climático en los ecosistemas forestales: integrando inventarios. Ecosistemas Revista Científica de Ecología y Medio Ambiente, 25(3): 51-59.

Granite Seed Co.

2014. The Granite Seed Catalog. Salt Lake City, Utah. USA. $112 \mathrm{p}$.

Hammermeister, A.

2000. Native seed harvesting and marketing. Native plant Society of Saskatchewan Inc. Saskaton, SK. Canada. 8 p.

Herrera, C.F.

2008. Caracterización sobre la calidad de semilla de tres variedades del pasto guinea Panicum maximum Jacq. bajo condiciones de producción del estado de Nayarit. XLIV Reunión Nacional de Investigación Pecuaria. Mérida, Yucatán. 121 p.

Ibarra, F.F.; Martin, M.R.F.; Denogean, B.; Moreno, S.M.; Salazar, A.L.

2009. Conveniencia del uso de semilla de pastos de origen local o de importación para la rehabilitación de agostaderos en el norte de México. XXII Congreso Internacional de Administración de Empresas Agropecuarias. Guadalajara, INEGI. Jalisco, México. 11 p.

2007. Mapa Digital de México. Sección Edafología. https://www. inegi.org.mx/temas/edafologia/ Consultado: 12/ago/2020.
INEGI.

2000. Síntesis de Información Geográfica del Estado de Sonora. Impreso. INEGI. 7 p.

Jump, A.S.; Mátyás, C.; Peñuelas, J.

2009 The altitude-for-latitude disparity in range retractions of woody species. Trends in Ecology and Evolution, 24(12): 694-701.

Jorgensen, K.R.; Stevens, R.

2004. Seed collection, cleaning, and storage, Chap. 24. En: Stephen B. M.; Stevens, R.; Shaw, N. (Comp.). Restoring Western Ranges and Wildlands. Gen. Tech. Rep. RMRSGTR-136-vol-3. U.S. Department of Agriculture, Forest Service, Rocky Mountain Research Station. Ft. Collins, Colorado. pp. 699-716.

Martín-Rivera, M.

2016. Impacto económico asociado con la cosecha de semilla de zámota y mezquite en la región central de Sonora, México. Revista Mexicana de Agronegocios, 38 (13452016-104516), 217-228.

Mc Caughey-Espinoza, M.; Ayala-Astorga, G.I.; VelázquezCaudillo, J.; Anaya-Islas, J.; Canseco-Vilchis, E.

2017. Creación de un jardín botánico y de árbol madre de arbustivas forrajeras nativas del estado de Sonora. Idesia (Arica), 35 (4): 35-45.

Mc-Caughey-Espinoza, D.; Reyes-Olivas, Á.; Ayala-Astorga, G.; Lugo-García, G.; Ochoa-Meza, A.; Pacheco-Olvera, A.

2019. Análisis químico proximal de Krameria erecta del Estado de Sonora. Revista Abanico Veterinario, 9(1): $1-12$.

Moritz, C.; Patton, J.L.; Conroy, C.J.; Parra, J.L. White, G.C. 2008. Impact of a century of climate change on small-mammal communities in Yosemite National Park, USA. Science, 322(5899): 261-264.

MP.

2011. The Statistical Discovery Software. SAS Institute Inc. Ver. 5.0.1 $1^{\mathrm{a}}$.

Padilla $\mathrm{H}$.

2018. Es Sonora el Estado con mayor deforestación. Periódico elimparcial. http://www.elimparcial.com/ EdicionEnLinea/Notas/Sonora/30062018/1352966 Consultado: 12/ago/2020.

Parmesan, C.

2006. Ecological and evolutionary responses to recent climate change. Annu. Rev. Ecol. Evol. Syst., 37: 637-669. 
Prentice, I.C.; Farquhar, G.D.; Fasham, M.J.R.; Goulden, M.L.; Heimann, M.; Jaramillo, V.J.; Kheshgi, H.S.; LeQuéré, C.; Scholes, R.J.; Wallace, D.W.

2001. The carbon cycle and atmospheric carbon dioxide. Cambridge University Press. pp. 135-237.

SAGARPA.

2010. Diagnóstico Sectorial Agropecuario, Pesquero y Recursos Naturales del Estado de Sonora. 52 p.

USDA-NRCS.

2015. United States Department of Agriculture. Coursetia glandulosa. Plants Database. http://plants.usda.gov/core/ profile? symbol=cogl8. Consultado: 12/ago/2020.
Vallentine, J.F.

1980. Range development and improvements. 2nd Ed. Brigham Young University Press. Provo, UT., USA. 545 pp.

Wilson, R.J.; Gutiérrez, D.; Gutiérrez, J.; Monserrat V. J.

2007. An elevational shift in butterfly species richness and composition accompanying recent climate change. Global Change Biology, 13(9): 1873-1887.

Yepes, A.; Buckeridge, M.S.

2011. Respuestas de las plantas ante los factores ambientales del cambio climático global (revisión). Colombia Forestal, 14(2): 213-232. 
